\title{
Re: Evaluation of Functional Outcomes after Laparoscopic Partial Nephrectomy Using Renal Scintigraphy: Clamped vs Clampless Technique
}

\author{
Francesco Porpiglia1, Riccardo Bertolo1, Daniele Amparore1, Valerio Podio2, Tiziana Angusti2, \\ Andrea Veltri2, Cristian Fiori1
}

1 University of Turin, San Luigi Gonzaga Hospital, Department of Urology, Turin, Italy

2San Luigi Gonzaga Hospital, Clinic of Radiology and Nuclear Medicine, Turin, Italy

BJU Int 2015;115:606-612. doi: 10.1111/bju.12834. Epub 2014 Oct 22.

\section{EDITORIAL COMMENT}

In the laparoscopic partial nephrectomy literature, the debate has mainly revolved around warm ischemia time. However, the effect of no ischemia or modest ischemia (less than 25-30 minutes) on renal function has not widely been evaluated yet.

In this retrospective study, the authors compared the on-clamp and off-clamp minimal invasive partial nephrectomy techniques on renal function in 87 kidney tumor patients. The authors analyzed functional outcomes by using renal scintigraphy at baseline and 3 months postoperatively. They carried out this study in T1c and T1b stage renal tumors with nearly normal split kidney function (45\% to 55\%). There were no differences in demographic, tumor or perioperative outcomes, including complications. The mean ischemia time of on-clamp technique was 18 minutes in 44 patients. The no ischemia group had a greater, although clinically insignificant, estimated blood loss (mean: 291 vs. $152 \mathrm{cc}$ ). The percentage reduction in renal scintigraphy values was not significantly different between the groups. The reductions in split renal function was 5\% in on-clamp group and 6\% in off-clamp group and $12 \%$ in on-clamp group and 17\% in off-clamp group regarding estimated renal plasmatic flow ( $p=0.587$ and $p=0.083$, respectively) at 3 months. Although they concluded that off-clamp partial nephrectomy was not harmful, there was no clinical advantage of off-clamp technique for the majority of patients according to their results. However, this study has not sufficient data to demonstrate the true final effect of warm ischemia time on renal functions because long-term effects of warm ischemia time on kidney functions are not investigated and need to be evaluated in further studies. 